\title{
MicroRNAs - diagnostic markers in Parkinson disease?
}

\section{6 \\ these studies hold promise for the use of peripheral miRNA biomarkers for the diagnosis of iPD}

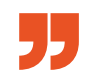

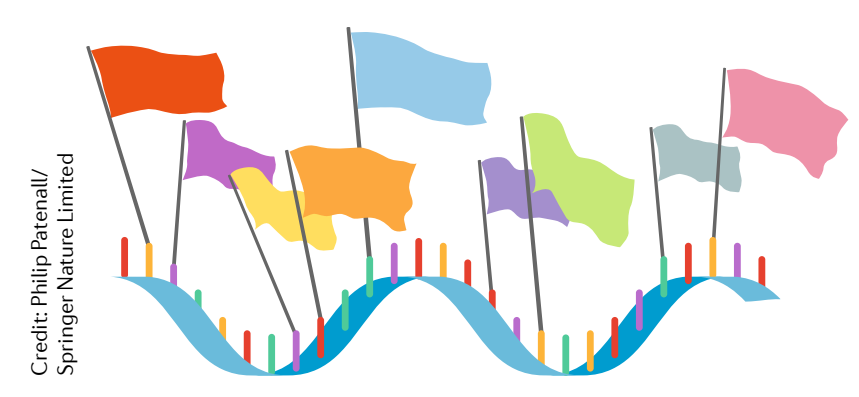

MicroRNA (miRNA) levels in the saliva and blood are altered in Parkinson disease (PD), two studies published in Movement Disorders have shown. The findings suggest that non-invasive miRNA-based tests could be used to diagnose PD and even different PD subtypes.

Currently, diagnosis of PD relies largely on clinical features, and no definitive diagnostic biomarkers have been identified. The new studies were prompted by previous work that suggested miRNAs that regulate genes and pathways associated with $\mathrm{PD}$ are altered in the disease.

The first study, led by Hyman Schipper, followed on from previous work by the same group with a mouse model of PD, in which haem oxygenase 1 (HO1) is overexpressed. In this model, miR-153 and miR-223 - two miRNAs that regulate the expression of $\alpha$-synuclein - are downregulated in the brain and the serum. Schipper and colleagues investigated whether these miRNAs could serve as diagnostic biomarkers of PD.

"We previously found significant elevation of $\mathrm{HO} 1$ protein in the saliva of patients with PD so we aimed to ascertain whether similar, downstream miRNA changes might be detectable in the saliva of humans with PD," explains Schipper. "We used

saliva because its collection is non-invasive and inexpensive."

The researchers collected saliva from 83 patients with PD and 77 controls with no neurological condition. The samples were analysed with reverse transcriptase quantitative PCR (RT-qPCR) to measure levels of miR-153 and miR-223. Salivary levels of both miRNAs were lower in patients with PD than in controls.

The findings suggest that salivary levels of miR-153 and miR-223 could be non-invasive, diagnostic markers of PD. "We now want to test salivary miR-153 and miR-223 levels in PD against neurological controls that include people with other neurodegenerative conditions, other synucleinopathies and other forms of parkinsonism to determine the specificity of the test for PD," says Schipper. "Another important objective is to look at salivary miR-153 and miR-223 in prodromal PD to assess how early in the course of the disease changes in miRNA levels become manifest."

In the other study, Epaminondas Doxakis and colleagues investigated miRNA markers of PD in idiopathic and genetic forms of the disease. The study built on increasing evidence that several miRNAs are involved in PD-related processes.

"Given that we have amassed substantial knowledge on the functions of these miRNAs in the brain, an obvious question was whether brain-enriched miRNAs are deregulated in PD," says Doxakis.

The study included 99 people with idiopathic PD (iPD), 53 with PD-causing mutations - 26 with mutations in SNCA, which encodes $\alpha$-synuclein, and 27 with mutations in $G B A$, which encodes glucocerebrosidase - and 101 healthy controls. Plasma samples were analysed with RT-qPCR to measure levels of 19 brain-enriched miRNAs and one ubiquitous miRNA that is abundant in the brain and targets $G B A$.

Several miRNAs were deregulated in all groups of patients with PD relative to healthy controls. "The deregulated miRNAs were different between idiopathic and genetic PD, but were very similar between the genetic forms of $\mathrm{PD}$, suggesting that $S N C A$ and GBA mutations converge on the same master miRNAs that drive the pathophysiological process in PD," explains Doxakis.

In silico analysis of the molecular pathways affected by the deregulated miRNAs pointed to the same conclusion, as the pathways converged for both genetic PD subtypes and iPD. The most prominent pathways identified were prion disease pathways and transforming growth factor- $\beta$, thyroid hormone and receptor tyrosine-protein kinase erbB signalling pathways.

"The findings of the two studies suggest that miRNA changes in PD, and likely other neurological diseases, are biofluid-specific," says Schipper. "Together, these studies hold promise for the use of peripheral miRNA biomarkers for the diagnosis of iPD."

Ian Fyfe

ORIGINAL ARTICLES Ravanidis, S. et al. Circulating brain-enriched microRNAs for detection and discrimination of idiopathic and genetic Parkinson's disease. Mov. Disord. https:// doi.org/10.1002/mds.27928 (2019)|Cressatti, M. et al. Salivary microR-153 and microR-223 levels as potential diagnostic biomarkers of idiopathic Parkinson's disease. Mov. Disord. https://doi.org/ 10.1002/mds.27935 (2019) 